\title{
Effects of CB2 Receptor Agonist JWH133 on Aß42-Induced Hippocampal Neurons
}

\author{
Jingfu Zhao ${ }^{1}$
}

\begin{abstract}
${ }^{1}$ Department of Physiology, School of basic medicine, Institute of Brain Science and Diseases, Qingdao University, Qingdao 266071, China

Abstract: Objective To observe the effects of Cannabinoid type 2 receptor (CB2R) on A $\beta$-induced hippocampal neuron. Methods Hippocampal neurons were pre-treated with $10 \mu \mathrm{M}$ JWH133 and then treated $100 \mathrm{nM}$ A $\beta 42$ oligomer for 7 day, the mRNA of Bcl-2 and Bax was measured by qRT-PCR, and the mitochondrial membrane potential and the production of ROS were measured by Flow Cytometry. Result A $\beta 42$ oligomer significantly decreased the ratio of $\mathrm{Bcl}-2 / \mathrm{Bax}$, and mitochondrial membrane potential, and increased the production of ROS. However, the pre-treatment JWH133 inhibited A 342 -induced the ratio of Bcl-2/Bax decrease, and increased the mitochondrial membrane potential, decreased the production of ROS. Conclusion Activation of CB2R could inhibit the effects of $A \beta 42$ on hippocampal neurons.
\end{abstract}

Keywords: Primary Hippocampal Neuron, Amyloid B-Protein, Cannabinoid Type 2 Receptor, JWH133

\begin{abstract}
1.Introduction
Alzheimer's disease (AD) is a common neurodegenerative disease in the elderly population, which seriously affects the middle-aged health, and there is a high incidence rate every year[1, 2]. The pathological features of $\mathrm{AD}$ are the large number of senile plaques in the brain and the formation of fiber tangles in neurons. Although the etiology and pathogenesis of AD have not yet been elucidated, many evidences indicated that amyloid $\beta$-protein $(\mathrm{A} \beta)$ toxicity, oxidative stress, neuroinflammation may result in damage of hippocampal neurons, which lead to the onset of $\mathrm{AD}[3,4]$.
\end{abstract}

$\mathrm{A} \beta$ toxicity is one of the main toxic mechanisms of $\mathrm{AD}$, A $\beta 42$ aggregates outside the cell to form oligomers, which could cause excessive phosphorylation of Tau protein in neurons, and also could bind to membrane receptors leads to increased intracellular oxidative stress $[5,6]$. At the same time, excessive accumulation of A $\beta 42$ oligomers leads to abnormal activation and dysfunction of immune cells[7, 8], resulting in excessive production of various inflammatory factors, causing neuroinflammation[9, 10].The expression of $\mathrm{Bcl}-2$ can protect neurons, and overexpression of $\mathrm{Bcl}-2$ can reduce the activity of caspase. But overexpression of Bax can inhibit the effect of bcl-2.

Cannabinoid type 2 receptor $(\mathrm{CB} 2 \mathrm{R})$ is a $\mathrm{G}$ protein coupled receptor, which is also expressed on hippocampal neurons[11, 12]. Unlike Cannabinoid type 1 receptor (CB1R), CB2R receptors are mainly expressed in the post-synaptic membrane. CB2R is mainly responsible for endogenous cannabinoid-mediated presynaptic inhibition. Under some pathological conditions, the expression of CB2R is up-regulated, which means that $\mathrm{CB} 2 \mathrm{R}$ may play a role in these neurological diseases[13]. The activation of CB2R of primary hippocampal neurons has a certain protective effect on neuronal hypoxic damage [14], which indicated that CB2R activation on hippocampal neurons could play a neuroprotective role. Meanwhile, treatment with $\mathrm{A} \beta$ for $\mathrm{CB} 2 \mathrm{R}$ knockout transgenic mice, the neuronal apoptosis was aggravated[15, 16]. So, could CB2R activation on hippocampal neurons inhibit $\mathrm{A} \beta$-induced hippocampal neuronal effects?

In the present study, we used $\mathrm{A} \beta 42$ oligomers to establish a model of primary hippocampal neuron apoptosis, and then pre-treated the neurons with CB2R selective agonist JWH133. Finally, we used qRT-PCR and Flow Cytometry to determine the effects of JWH133.

\section{Materials and methods}

2.1 primary hippocampal neuron culture and treatment

Primary hippocampal neuronal medium

$490 \mathrm{~mL}$ of Neurobasal-A medium, $10 \mathrm{~mL}$ B27, $1 \mathrm{~mL}$ of cyan/streptomycin solution were mixed.

Primary hippocampal neuron culture and treatment The culture dishes were treated with $100 \mu \mathrm{g} / \mathrm{mL}$ poly-D-lysine. Before the experiment, wash the culture dishes with double distilled water. Primary hippocampal neuron was obtained from newborn SD rats within 24 hours and suspended in a Neurobasal-A medium with B27, and then inoculated into a culture 
dishes pre-plated with poly-D-lysine, and placed at $37^{\circ} \mathrm{C}, 5 \% \mathrm{CO} 2$ incubator. Incubated in the incubator for about 7 days, and the neurons were treated in groups when the cell density reached $80 \%$, as follows: Control group: treated with Neurobasal-A with B27 for 7 days;

$\mathrm{A} \beta$ group: incubated with $100 \mathrm{nM} \mathrm{A} \beta 42$ oligomers for 7 days;

A $\beta+J W H 133$ group: incubated with $10 \mu \mathrm{M}$ JWH133 and $100 \mathrm{nM} A \beta 42$ oligomers for 7 days;

$\mathrm{A} \beta+\mathrm{JWH} 133+\mathrm{AM} 630$ group: incubated with $10 \mu \mathrm{M}$

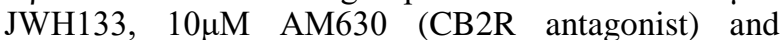
$100 \mathrm{nM} A \beta 42$ oligomers for 7 days.

\subsection{Real time fluorescence quantitative polymerase chain reaction (qRT-PCR)}

Total RNA was isolated from the primary hippocampal neuron using the Trizol reagent. Follow Thermo's Reverse Transcription Kit, the illustrated procedure is reverse transcribed. Then the cDNA is amplified. Proceed as follows primer sequences:

Bcl-2

forward: 5'-TGTGGATGACTGACTACCTGAACC-3 reverse: 5'-CAGCCAGGAGAAATCAAACAGAGG-3' Bax

forward: 5'-CGGCGAATTGGAGATGAACTGG-3' reverse: 5'-CTAGCAAAGTAGAAGAGGGCAACC-3' GAPDH

Forward: 5'-GGCACAGTCAAGGCTGAGAATG-3' reverse: 5'-ATGGTGGTGAAGACGCCAGTA-3'

\subsection{Flow Cytometry}

Detect changes in mitochondrial membrane potential (MMP):

After treatment for $14 \mathrm{~d}$, remove the culture medium, add Rhodamine 123 to each well. Incubated at $37{ }^{\circ} \mathrm{C}$ in the dark for $30 \mathrm{~min}$. and washed in cold Hepes buffered saline (HBS) twice. The MMP of primary hippocampal neuron were analyzed using flow cytometry.

Detect changes in reactive oxygen species (ROS):

After treatment for $14 \mathrm{~d}$, remove the culture medium, add 2,7-Dichlorofluorescin diacetate to each well. Incubated at $37{ }^{\circ} \mathrm{C}$ in the dark for $30 \mathrm{~min}$. and washed in cold Hepes buffered saline (HBS) twice. The production of ROS of primary hippocampal neuron were analyzed using flow cytometry.

\subsection{Statistical analysis}

Data are presented as mean \pm SEM with number of samples (n). A probability level of $\mathrm{p}<0.05$ was considered to be statistically significant. Significant differences were determined using the two-tailed Student's t-test or one-way ANOVA as appropriate.

\section{Results}

3.1 CB2R agonist, JWH-133 against the A $\beta 42$-induced reduction of $\mathrm{Bcl}-2 / \mathrm{Bax}$ ratio in hippocampal neuronal cultures

We evaluate the roles of JWH133 in the A $\beta 42$-induced alterations of $\mathrm{Bcl}-2 / \mathrm{Bax}$ ratios. $\mathrm{qRT}$-PCR showed that the $\mathrm{Bcl}-2 / \mathrm{Bax}$ ratios in the group of control, compared to control group, the $\mathrm{Bcl}-2 / \mathrm{Bax}$ ratio decreased in the $\mathrm{A} \beta 42$ group $(P<0.001)$; However, the $\mathrm{Bcl}-2 / \mathrm{Bax}$ ratio in the $\mathrm{A} \beta 42+\mathrm{JWH} 133$ group was significantly higher than that in the $\mathrm{A} \beta 42$ alone $\operatorname{group}(P<0.01)$; The $\mathrm{Bcl}-2 / \mathrm{Bax}$ ratio in the $\mathrm{A} \beta 42+\mathrm{AM} 630+\mathrm{JWH} 133$ group was significantly lower than that in the $A \beta 42+$ JWH133 group $(P<0.05)$ (Fig1).

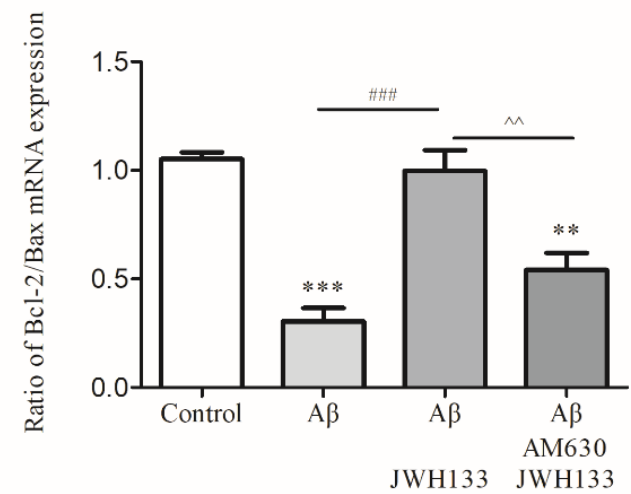

Fig 1, JWH-133 against the A $\beta 42$-induced decrease of Bcl-2/Bax ratio in hippocampal neuronal cultures.

(A)Raw data shows the Western-blot results of $\mathrm{Bcl}-2 / \mathrm{Bax}$ ratio in control, $\mathrm{A} \beta 42, \mathrm{~A} \beta 42+\mathrm{JWH} 133$, and $\mathrm{A} \beta 42+\mathrm{AM} 630+\mathrm{JWH} 133$ groups. (B)Bar graph summarizes 4 groups of experiments, and showed that JWH133 prevented A $\beta 42$-induced decrease of Bcl-2/Bax ratio, which could be abolished by AM630.

3.2 CB2R agonist, JWH-133 against the A $\beta 42$-induced reduction of Mitochondrial membrane potential in hippocampal neuronal cultures.

Flow Cytometry is used to detect the change in mitochondrial membrane potential in the hippocampal neuron apoptosis model induced by $\mathrm{A} \beta 42$ and whether it could be suppressed by the JWH133 treatment. The results showed that compared with the control group, the mitochondrial membrane potential of the $A \beta 42$ group decreased significantly $(P<0.01)$, and the A $342+$ JWH133 group mitochondrial membrane potential increased compared with the $A \beta 42$ group $(P<0.05)$. Compared with $\mathrm{A} \beta+\mathrm{JWH} 133$ group, A $\beta+$ AM630+JWH133 group had significantly lower mitochondrial membrane potential $(P<0.01)$ (Fig 2$)$.
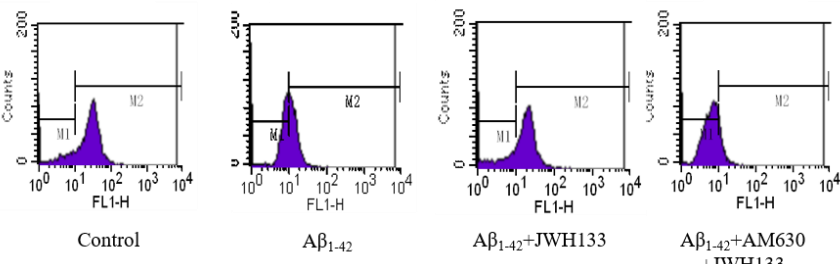

$\mathrm{A} \beta_{1-42}$

$\mathrm{A} \beta_{1-42}+\mathrm{JWH} 133$ $+\mathrm{JWH} 133$ 


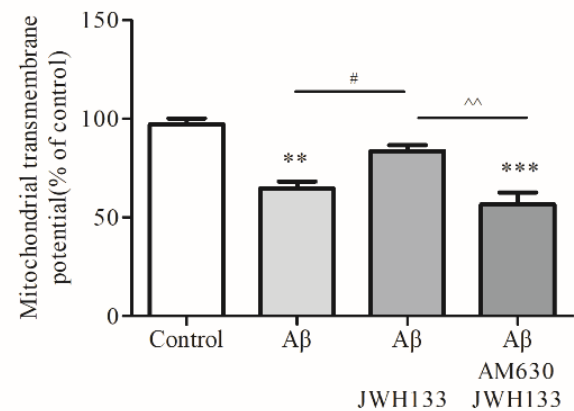

Fig2. JWH133 inhibits A $\beta$ induced mitochondrial membrane potential changes in hippocampal neurons (A) Representatives of the fluorometric assay on mitochondrial membrane potential of hippocampal neurons in four treatment groups. (B) A $\beta 42$ treatment could decrease mitochondrial membrane potential of hippocampal neurons, which could be antagonized by JWH133. Data represents the mean \pm S.E.M of 3 independent experiments $(* * \mathrm{P}<0.01 ; * \mathrm{P}<0.05$; $\# \mathrm{P}<0.05$; $\wedge \wedge \mathrm{P}<0.01)$.

\subsection{CB2R agonist, JWH133 against the} A 342 -induced increase of ROS production in hippocampal cultures.

We applied Flow Cytometry to detect the effect of JWH133 on the changes of reactive oxygen species (ROS) levels in hippocampal neurons injured by $A \beta 42$. The results showed that $A \beta 42$ chronically treated hippocampal neurons had significantly higher ROS production compared with the control group $(P<0.01)$, $\mathrm{A} \beta+\mathrm{JWH} 133$ had lower ROS production than the $\mathrm{A} \beta$ group $(P<0.05)$, and The production of ROS in $\mathrm{A} \beta+$ AM630+JWH133 group decreased $(P<0.05)$ (Fig 3$)$.
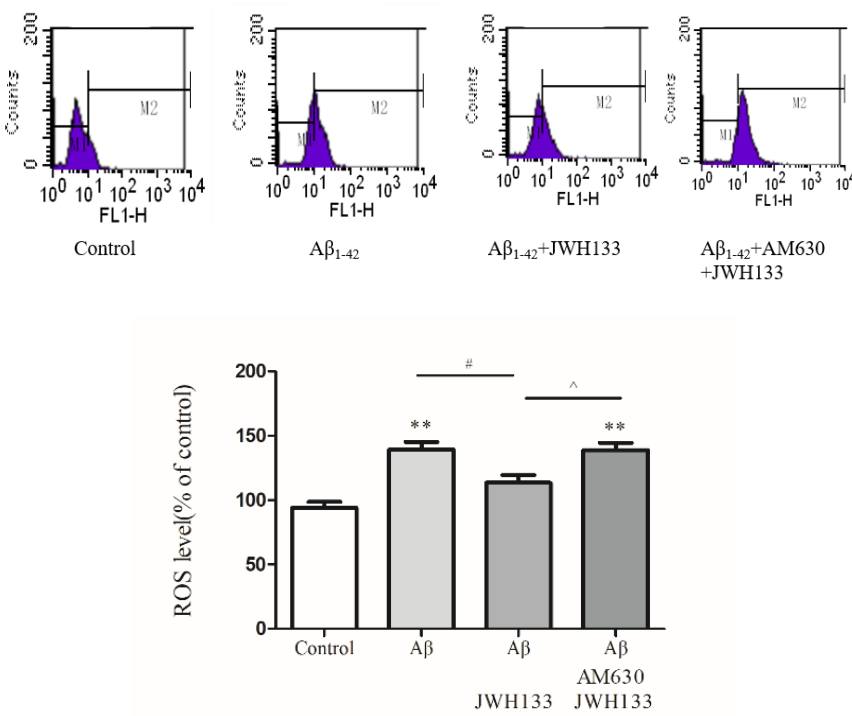

Fig 3. JWH133 inhibits A $\beta 1-42$ induced ROS changes in hippocampal neurons

(A) Representatives of the fluorometric assay on ROS of hippocampal neurons in four treatment groups. (B)
Statistical analysis of ROS. A $\beta 42$ treatment could increase the production of ROS of hippocampal neurons, which could be antagonized by JWH133. Data represents the mean \pm S.E.M of 3 independent experiments $(* * \mathrm{P}<0.01 ; * \mathrm{P}<0.05 ; \# \mathrm{P}<0.05 ; \wedge \mathrm{P}<0.05)$.

\section{Discussion}

The major and new finding of this study is that the activation of hippocampal CB2Rs could inhibit $\mathrm{A} \beta 42$-induced decrease of $\mathrm{Bcl}-2 / \mathrm{Bax}$ ratio and the mitochondrial membrane potential, and could inhibited the increased of ROS production in hippocampal neurons. Bcl-2 can inhibit the occurrence of apoptosis by inhibiting the release of cytochrome $c[17,18]$. Bax inhibits its protective effect by binding to $\mathrm{Bcl} 2[19]$.

A $\beta 42$ oligomers play a very important role in neuronal degeneration and $\mathrm{AD}[20,21]$, but the mechanisms are still unclear. Therefore, understanding of such mechanisms are still to help improve $\mathrm{AD}$ treatment. Our results showed that $A \beta 42$ could decreased the ratio of $\mathrm{Bcl}-2 / \mathrm{Bax}$, the mitochondrial membrane potential, and increased the production of ROS.

CB2R is a $\mathrm{G}$ protein-coupled receptor that was found in 1993[22]. More and more scholars have proved that $\mathrm{CB} 2 \mathrm{R}$ is also expressed in neurons. CB2R show neuroprotective effects in the pathogenesis of $\mathrm{AD}$. For example, on the glial cells around the senile plaques, the increased expression of CB2R of glial cells is related to the level of $A \beta 42$ and its deposition[23]. Increased expression and activation of CB2R enhances the ability of macrophages to clear $A \beta 42[24]$.

The reduction of mitochondrial membrane potential promotes the cell to enter the irreversible apoptosis process[25], mainly because the reduction of mitochondrial membrane potential causes the release of cytochrome $\mathrm{C}$ into the cytosol, thereby activating the cascade reaction of apoptosis effector Caspase[26]. At the same time, the decrease in mitochondrial membrane potential also leads to an increase in the production of ROS[27]. Our results showed that after pre-treatment with CB2R agonist JWH133, A 442 -induced the decrease of the ratio of $\mathrm{Bcl}-2 / \mathrm{Bax}$ was inhibited, and activated $\mathrm{CB} 2 \mathrm{R}$ increased mitochondrial membrane potential and decreased the production of ROS. This effect of JWH133 can be inhibited by CB2R antagonist AM630.

Toxicity of $A \beta 42$ is closely related to $A D$, so activation of $\mathrm{CB} 2 \mathrm{R}$ can inhibit A $\beta 42$-induced pathological changes in neurons. However, the underlying mechanisms of the effect of $\mathrm{CB} 2 \mathrm{R}$ on $\mathrm{A} \beta 42$-induced neurotoxicity should be further studied in the future.

\section{Acknowledgments and Fundings}

This work was supported by grants from National Natural Science Foundation of China (81771437) 


\section{References}

1. Lane, C.A., J. Hardy, and J.M. Schott, Alzheimer's disease. Eur J Neurol, 2018. 25(1): p. 59-70.

2. Bondi, M.W., E.C. Edmonds, and D.P. Salmon, Alzheimer's Disease: Past, Present, and Future. J Int Neuropsychol Soc, 2017. 23(9-10): p. 818-831.

3. Mantzavinos, V. and A. Alexiou, Biomarkers for Alzheimer's Disease Diagnosis. Curr Alzheimer Res, 2017. 14(11): p. 1149-1154.

4. Galla, L., et al., Intracellular Calcium Dysregulation by the Alzheimer's Disease-Linked Protein Presenilin 2. Int J Mol Sci, 2020. 21(3).

5. Cheignon, C., et al., Oxidative stress and the amyloid beta peptide in Alzheimer's disease. Redox Biol, 2018. 14: p. 450-464.

6. Bloom, G.S., Amyloid- $\beta$ and tau: the trigger and bullet in Alzheimer disease pathogenesis. JAMA Neurol, 2014. 71(4): p. 505-8.

7. Kowalski, K. and A. Mulak, Brain-Gut-Microbiota Axis in Alzheimer's Disease. J Neurogastroenterol Motil, 2019. 25(1): p. 48-60.

8. Gosztyla, M.L., H.M. Brothers, and S.R. Robinson, Alzheimer's Amyloid- $\beta$ is an Antimicrobial Peptide: A Review of the Evidence. J Alzheimers Dis, 2018. 62(4): p. 1495-1506.

9. Nazem, A., et al., Rodent models of neuroinflammation for Alzheimer's disease. J Neuroinflammation, 2015. 12: p. 74.

10. Zhang, P., et al., Senolytic therapy alleviates A $\beta$-associated oligodendrocyte progenitor cell senescence and cognitive deficits in an Alzheimer's disease model. Nat Neurosci, 2019. 22(5): p. 719-728.

11. Stempel, A.V., et al., Cannabinoid Type 2 Receptors Mediate a Cell Type-Specific Plasticity in the Hippocampus. Neuron, 2016. 90(4): p. 795-809.

12. $\mathrm{Wu}, \mathrm{Q}$. and $\mathrm{H}$. Wang, The spatiotemporal expression changes of $\mathrm{CB} 2 \mathrm{R}$ in the hippocampus of rats following pilocarpine-induced status epilepticus. Epilepsy Res, 2018. 148: p. 8-16.

13. Wu, J., Cannabis, cannabinoid receptors, and endocannabinoid system: yesterday, today, and tomorrow. Acta Pharmacol Sin, 2019. 40(3): p. 297-299.

14. Ward, S.J., et al., Surprising outcomes in cannabinoid CB1/CB2 receptor double knockout mice in two models of ischemia. Life Sci, 2018. 195: p. 1-5.
15. Wang, L., et al., Deletion of Type-2 Cannabinoid Receptor Induces Alzheimer's Disease-Like Tau Pathology and Memory Impairment Through AMPK/GSK3 $\beta$ Pathway. Mol Neurobiol, 2018. 55(6): p. 4731-4744.

16. $\mathrm{Li}, \mathrm{C}$., et al., CB2 cannabinoid receptor agonist ameliorates novel object recognition but not spatial memory in transgenic APP/PS1 mice. Neurosci Lett, 2019. 707: p. 134286.

17. Siddiqui, W.A., A. Ahad, and H. Ahsan, The mystery of BCL2 family: Bcl-2 proteins and apoptosis: an update. Arch Toxicol, 2015. 89(3): p. 289-317.

18. Kontos, C.K., M.I. Christodoulou, and A. Scorilas, Apoptosis-related BCL2-family members: Key players in chemotherapy. Anticancer Agents Med Chem, 2014. 14(3): p. 353-74.

19. Reyna, D.E., et al., Direct Activation of BAX by BTSA1 Overcomes Apoptosis Resistance in Acute Myeloid Leukemia. Cancer Cell, 2017. 32(4): p. 490-505.e10.

20. Blennow, K. and H. Zetterberg, Biomarkers for Alzheimer's disease: current status and prospects for the future. J Intern Med 2018. 284(6): p. 643-663.

21. Rosenberg, R.N., et al., Genomics of Alzheimer Disease: A Review. JAMA Neurol, 2016. 73(7): p. 867-74.

22. Zhou, H., et al., Human Cannabinoid Receptor 2 Ligand-Interaction Motif: Transmembrane Helix 2 Cysteine, C2.59(89), as Determinant of Classical Cannabinoid Agonist Activity and Binding Pose. Acs Chemical Neuroscience, 2017: p. acschemneuro.7b00003.

23. Leinwand, K.L., et al., Cannabinoid Receptor-2 Ameliorates Inflammation in Murine Model of Crohn's Disease. Journal of Crohns \& Colitis, 2017. 11(11): p. 1369.

24. Solas, M., et al., CB2 receptor and amyloid pathology in frontal cortex of Alzheimer's disease patients. Neurobiol Aging, 2013. 34(3): p. 805-8.

25. Ly, J.D., D.R. Grubb, and A. Lawen, The mitochondrial membrane potential $(\Delta \psi \mathrm{m})$ in apoptosis; An update. Apoptosis An International Journal on Programmed Cell Death, 2003. 8(2): p. 115-128.

26. Jurgensmeier, et al., Bax directly induces release of cytochrome c from isolated mitochondria. Proceedings of the National Academy of Sciences of the United States of America, 1998.

27. Bedard, et al., The NOX Family of ROS-Generating NADPH Oxidases: Physiology and Pathophysiology. Physiological Reviews, 2007. 